\title{
Effets du niveau alimentaire sur la saisonnalité de l'activité sexuelle chez la brebis Timahdite : influence de la leptine et du système IGF
}

\author{
B. Archa ${ }^{1}$ M. Chentouf ${ }^{2}$ J.L. Bister ${ }^{3 *}$
}

\begin{abstract}
Mots-clés
Ovin - Niveau d'alimentation Reproduction - Périodicité - Saison Protéine sanguine - Facteur de croissance IGF - Maroc.
\end{abstract}

\begin{abstract}
Résumé
Les effets du niveau alimentaire sur la saisonnalité de la reproduction ont été étudiés chez la brebis Timahdite dans la région du Moyen Atlas du Maroc $\left(33^{\circ} \mathrm{N}\right)$. Des brebis adultes (3-4 ans), non gravides et non allaitantes, ont été réparties en deux lots, $\mathrm{NH}(\mathrm{n}=10)$ et $\mathrm{NB}(\mathrm{n}=9)$, en fonction de régimes alimentaires correspondant respectivement à deux et une fois leurs besoins d'entretien. Durant une année, les chaleurs ont été observées deux fois par jour et des prises de sang ont été réalisées chaque semaine pour le dosage des niveaux plasmatiques en progestérone, et en facteurs de croissance comme la leptine, I'IGF-1 et ses protéines de liaison (IGFBP-2 et IGFBP-3). Le niveau alimentaire élevé a stimulé la fonction reproductrice des brebis $\mathrm{NH}$, puisqu'elles ont présenté une saison de reproduction plus longue et ont manifesté un nombre de cycles normaux significativement plus élevé que les brebis du lot NB $(7,8 \pm 1,4$ vs 3,9 $\pm 1,9 ; \mathrm{P}<0,05)$. La leptine, I'IGF-1 et l'IGFBP-2 ont semblé impliqués dans cette stimulation de l'activité reproductrice. Les brebis $\mathrm{NH}$ ont présenté des teneurs plasmatiques significativement plus élevées que celles des brebis NB en leptine $(3,79 \pm 0,38$ vs $3,30 \pm 0,29 \mathrm{ng} / \mathrm{mL} ; \mathrm{P}<0,05)$ et en IGF-1 (76 \pm 28 vs $58 \pm 21 \mathrm{ng} / \mathrm{mL} ; \mathrm{P}<0,01)$, alors que la teneur plasmatique en IGFBP-2 a été significativement plus élevée chez les brebis NB (99 \pm 24 vs $68 \pm 21$ p. 100, $\mathrm{P}<0,01)$. En revanche, aucune différence significative n'a été observée pour la teneur en IGFBP-3 (103 \pm 52 vs $96 \pm 17$ p. $100 ; \mathrm{P}>0,05)$. Ce travail a permis de conclure que la brebis Timahdite présentait une activité reproductrice saisonnière modulée par l'alimentation.
\end{abstract}

\section{INTRODUCTION}

Chez les animaux domestiques, la productivité peut être limitée par la saisonnalité de la reproduction. Certaines races de brebis ou de chèvres montrent une variation saisonnière de leur activité de reproduction durant l'année $(22,35,45)$. Chez les petits ruminants,

\footnotetext{
1. Département des productions animales et du pastoralisme, Ecole nationale d'agriculture, Meknès, Maroc.

2. Inra, unité de recherches Productions animales, Tanger, Maroc.

3. Laboratoire de physiologie animale, Faculté universitaire Notre-Dame de la Paix, Fundp, 61 rue de Bruxelles, 5000 Namur, Belgique.

* Auteur pour la correspondance

Tél. : +32 81724375 ; fax : +32 81724420

E-mail : Jean-loup.Bister@fundp.ac.be
}

il est bien connu que cette saisonnalité est sous le contrôle photopériodique avec des jours croissants inhibiteurs et des jours décroissants stimulateurs de l'activité sexuelle (19). Cependant, d'autres paramètres environnementaux, tels que la disponibilité alimentaire et les interactions sociales (20), seraient vraisemblablement des régulateurs potentiels de la saisonnalité de la reproduction.

La nutrition est considérée comme un facteur important affectant la fonction de reproduction chez les ruminants domestiques (11). Chez le mouton, elle affecte l'âge à la puberté, la fertilité, le taux d'ovulation et la survie de l'embryon (2). Plusieurs facteurs métaboliques sont impliqués dans la régulation de la fonction ovarienne. Ceux-ci incluent des hormones et des facteurs de croissance, tels que la leptine et les facteurs de croissance apparentés à l'insuline - 1 (IGF-1) et leurs protéines de liaison (41). 
La leptine, principalement produite par les adipocytes, serait le messager informant le système nerveux central sur le niveau de réserves corporelles $(6,7)$. Des récepteurs de la leptine ont été détectés au niveau de l'hypothalamus et de la glande hypophysaire indiquant son action à ce niveau (8). Chez la vache, l'inhibition de la sécrétion d'hormone lutéinisante (LH) par la restriction alimentaire peut être inversée par un traitement avec la leptine, montrant une association positive entre la sécrétion de LH et la leptine (1). Le système des IGF hépatiques joue un rôle important dans la différenciation et la croissance cellulaire et constitue le médiateur principal de l'hormone de croissance (37). La libération des IGF par leurs protéines porteuses (IGFBP) est contrôlée par l'action des protéases d'IGFBP spécifiques (30). L'IGF-1, qui est aussi sécrété cellules de la granulosa (25), stimule le développement folliculaire en agissant en synergie avec l'hormone folliculostimulante (FSH) au niveau des follicules dominants chez la vache (14). L'IGF-1 stimule aussi la prolifération des cellules de la granulosa au niveau des petits follicules antraux chez le mouton (27) et stimule la sécrétion stéroïdienne par les cellules de la granulosa des grands follicules ovins $(28,4)$. Il y a une relation certaine entre la variation des concentrations d'IGF-1, induite par la nutrition, et l'activité ovarienne (42).

L'objectif de ce travail a été d'étudier l'incidence du niveau alimentaire sur la saisonnalité de l'activité sexuelle, sur la croissance corporelle et sur les niveaux plasmatiques de leptine, d'IGF-1 et des IGFBP chez les brebis de race Timahdite.

\section{MATERIEL ET METHODES}

\section{Animaux et traitements}

L'étude a été conduite à la ferme d'application de l'Ecole nationale d'agriculture de Meknès (335' N, $5^{\circ} 32^{\prime} \mathrm{O}$ ). Dix-neuf brebis Timahdite adultes (3-4 ans), non gravides et non allaitantes, ont été hébergées dans une bergerie en conditions de lumière naturelle pendant une année. Les animaux ont été répartis en deux groupes de poids moyens similaires $(30 \pm 4 \mathrm{~kg})$ et affectés aléatoirement à deux niveaux alimentaires : un niveau bas (NB) calculé pour couvrir les besoins d'entretien $(\mathrm{n}=9)$ et un niveau haut $(\mathrm{NH})$ égal au double des besoins d'entretien $(n=10)$. Le calcul de la ration a été basé sur les recommandations de l'Institut national de la recherche agronomique (17). La composition de la ration des deux groupes d'animaux est présentée dans le tableau I.

\section{Tableau I}

Composition des rations alimentaires des deux groupes d'animaux

\begin{tabular}{lcc} 
& Niveau bas & Niveau haut \\
$\begin{array}{l}\text { Ration quotidienne } \\
\text { par animal }\end{array}$ & $\begin{array}{c}\mathbf{9 1 0} \mathbf{g} \text { paille de blé } \\
\mathbf{1 1 0} \mathbf{g} \text { TT } \\
\text { et } \mathbf{1 1} \mathbf{g} \text { CMV }\end{array}$ & $\begin{array}{c}\mathbf{9 1 0} \mathbf{g} \text { paille de blé } \\
\mathbf{4 1 0} \mathbf{g} \text { d'orge, } \\
\mathbf{1 1 5} \mathbf{g} \text { TT et } \mathbf{3 9} \mathbf{g} \text { CMV }\end{array}$ \\
\hline UFL/j/animal & 0,41 & 0,82 \\
MAD (g/j/animal) & 32 & 64 \\
Ca (g/j/animal) & 3 & 6 \\
P (g/j/animal) & 2,5 & 5
\end{tabular}

TT : tourteau de tournesol ; CMV: complément minéral vitaminique ; UFL : unité fourragère lait ; MAD : matière azotée digestible ; $\mathrm{Ca}$ : calcium ; $\mathrm{P}$ : phosphore
Les chaleurs ont été détectées deux fois par jour à l'aide d'un bélier vasectomisé muni d'un marqueur. Toute brebis qui est restée immobile et a accepté le chevauchement du bélier a été considérée en chaleurs, et l'observation d'une nette coloration au niveau de la croupe a été requise. Des pesées mensuelles de tout le troupeau ont été réalisées. Tous les animaux ont été vaccinés contre l'entérotoxémie et traités contre les parasites internes et externes.

\section{Prises de sang}

Des prises de sang hebdomadaires au niveau de la veine jugulaire ont été réalisées à l'aide de tubes sous vide héparinés. Le plasma a été séparé par centrifugation, à $2000 \mathrm{~g}$ pendant $15 \mathrm{~min}$, et stocké et congelé à $-20{ }^{\circ} \mathrm{C}$ jusqu' au moment des dosages radio-immunologiques (RIA) de la progestérone (P4), de la leptine, de l'IGF-1 et des IGFBP. Les dosages de la P4 ont concerné les cinquante et une prises de sang effectuées, et ceux de l'IGF-1 et de la leptine ont consisté en une prise de sang par mois pour tous les animaux. Enfin, les analyses des IGFBP ont porté sur six échantillons par animal choisis sur trois périodes : début, milieu et fin de la période d'essai.

\section{Analyses hormonales}

Les concentrations de la P4 ont été évaluées par la méthode RIA avec double anticorps, comme décrit par Bister et Paquay (5). Un taux de $\mathrm{P} 4$ supérieur ou égal à $1 \mathrm{ng} / \mathrm{mL}$ a été considéré comme le signe d'un corps jaune actif et donc d'une ovulation. La durée moyenne du cycle œstral est définie comme étant le nombre de jours entre deux détections de chaleurs. La durée moyenne de la saison d'anœstrus est définie comme le nombre de jours entre la dernière détection de chaleurs d'une saison de reproduction donnée et la première détection de chaleurs de la saison suivante (12). Trois catégories de cycles sexuels ont été définies : un cycle normal si les chaleurs ont été accompagnées d'une ovulation, un cycle à ovulation silencieuse si l'ovulation n'a pas été associée à un comportement œstral, et un cycle à œstrus anovulatoire si la détection de chaleurs n'a pas été accompagnée d'une ovulation.

Les concentrations plasmatiques de leptine ont été déterminées à l'aide du kit RIA multi-espèces (XL-85K, Linco Research, USA). Le seuil de détection minimal du dosage est de $1,0 \mathrm{ng} / \mathrm{mL}$ équivalent humain et les coefficients de variation de la sensibilité intra- et inter-dosage sont respectivement de 3,4 et 8,7 p. 100. Le dosage d'IGF-1 a été réalisé par la méthode RIA selon le protocole développé par Renaville et coll. (33) chez les bovins. Le seuil de détection est de $1 \mathrm{ng} / \mathrm{mL}$ et les coefficients de variation intra et inter-dosage sont respectivement de 12 et 16 p. 100 pour les faibles concentrations de la courbe standard $(2,5 \mathrm{ng} / \mathrm{mL})$ et 6,5 et 9 p. 100 , respectivement, pour les fortes concentrations de la courbe standard $(50 \mathrm{ng} / \mathrm{mL})$. La détection des IGFBP dans les échantillons de plasma a été réalisée par la procédure du western ligand blot selon la méthode décrite par Renaville et coll. (33) chez les bovins.

\section{Analyses statistiques}

Les données ont été exprimées sous forme de moyenne ( \pm écart type) de tous les animaux du même groupe. L'analyse statistique a été effectuée avec les procédures du modèle linéaire généralisé (GLM) et l'Anova du SAS (36). La comparaison des moyennes a été réalisée à l'aide du test statistique $t$ de Student. L'analyse de corrélation de Pearson et la régression linéaire ont été réalisées pour évaluer de possibles corrélations entre le poids, le pourcentage des brebis en chaleurs et les concentrations d'hormones (leptine, IGF-1, IGFBP). La signification statistique a été acceptée avec $\mathrm{P}<0,05$. 


\section{RESULTATS}

\section{Effet du niveau alimentaire sur la saisonnalité de l'activité sexuelle}

La figure 1 montre que l'activité sexuelle a été influencée à la fois par la photopériode et le niveau alimentaire. Elle a été stimulée par la diminution de la durée du jour et inhibée par l'augmentation de la durée du jour. Toutefois, l'effet dépressif d'une photopériode croissante a été moins marqué chez les brebis NH que chez les brebis NB. En effet, les brebis NB ont arrêté complètement de manifester des chaleurs à partir du mois de janvier, alors que chez les brebis $\mathrm{NH}$, la moitié des animaux (5/10) ont continué de manifester des chaleurs durant les mois de février et mars, un peu moins en avril (4/10) et avec un minimum en mai (3/10). Si l'on considère qu'une saison moyenne correspond à la période pendant laquelle au moins la moitié des femelles présentent des cycles (estimation d'après la manifestation de chaleurs), elle a été plus longue chez les brebis NH (juillet à mars) que chez les brebis NB (août à décembre).

En moyenne, par brebis, le nombre total de cycles, toutes catégories comprises, et de cycles œstraux normaux a été plus élevé chez les brebis NH que chez les brebis NB $(\mathrm{P}<0,005)$. Cependant, les deux lots de brebis ont présenté le même nombre moyen de cycles anormaux $(\mathrm{P}>0,05$; tableau II).

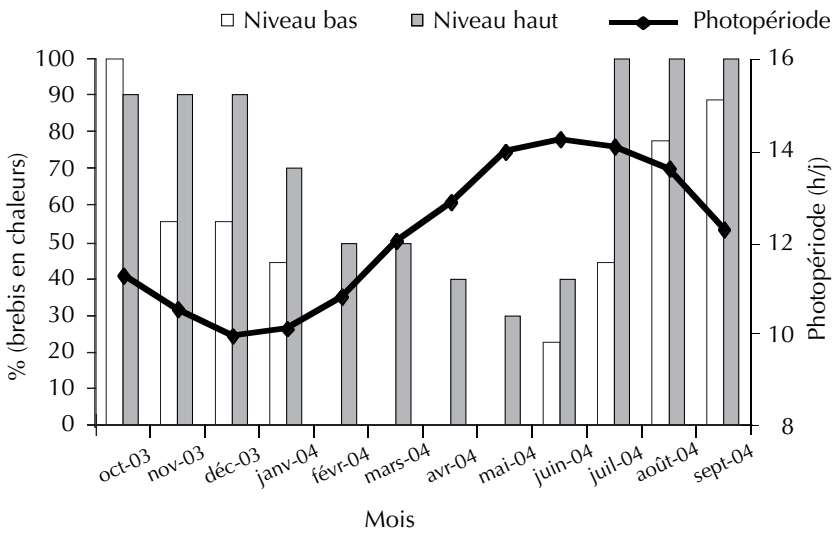

Figure 1 : fluctuations de la photopériode et histogramme des fréquences des brebis en chaleurs en fonction de leur niveau alimentaire bas $(n=9)$ et haut $(n=10)$.

\section{Tableau II}

Nombre moyen \pm écart-type des différents cycles en fonction du niveau alimentaire

\begin{tabular}{lcccc} 
Lot & $\begin{array}{c}\text { Total } \\
\text { cycles }\end{array}$ & $\begin{array}{c}\text { Cycles } \\
\text { ostraux } \\
\text { normaux }\end{array}$ & $\begin{array}{c}\text { OEstrus } \\
\text { anovulatoires }\end{array}$ & $\begin{array}{c}\text { Ovulations } \\
\text { silencieuses }\end{array}$ \\
\hline $\begin{array}{l}\text { NB } \\
(n=9)\end{array}$ & $7,0 \pm 2,7^{b}$ & $3,9 \pm 1,9$ b & $2,2 \pm 1,2$ & $0,9 \pm 0,8$ \\
NH & $11,2 \pm 1,9^{a}$ & $7,8 \pm 1,4^{a}$ & $2,5 \pm 1,1$ & $0,9 \pm 1,0$ \\
$(n=10)$ & & & & \\
\hline
\end{tabular}

$\mathrm{NB}$ : niveau alimentaire bas ; $\mathrm{NH}$ : niveau alimentaire haut

a,b Les valeurs de la même colonne portant en exposant des lettres différentes sont significativement différentes $(\mathrm{P}<0,05)$

\section{Effet du niveau alimentaire sur le poids vif}

La courbe de croissance a montré que le niveau alimentaire affectait significativement l'évolution du poids moyen des animaux (figure 2). Au début de l'essai, les deux lots de brebis affichaient un poids moyen similaire de $29,2 \pm 4,9 \mathrm{~kg}$ et $30,9 \pm 3,6 \mathrm{~kg}$. En fin d'essai, le poids moyen des brebis NB a été significativement plus bas que celui des brebis $\mathrm{NH}(28,0 \pm 5,1 \mathrm{~kg}$ vs $35,2 \pm 4,7 \mathrm{~kg} ; \mathrm{P}<$ $0,05)$. Il est à noter qu'une corrélation positive a été calculée entre le pourcentage des brebis en chaleurs et le poids vif des brebis $(\mathrm{r}=$ $0,50 ; \mathrm{P}<0,05)$

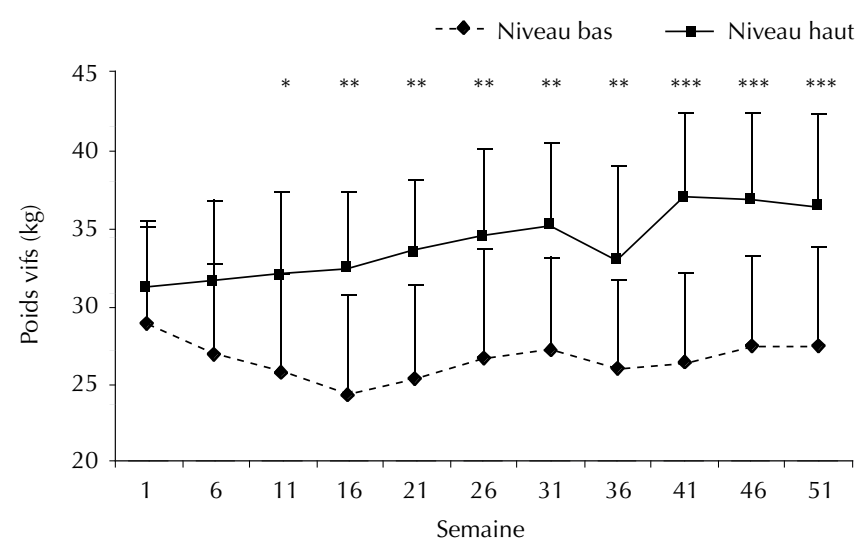

Figure 2 : évolution du poids vif moyen des brebis à niveau alimentaire bas $(n=9)$ et des brebis à niveau alimentaire haut $(n=10)$.

* $P<0,05$; ${ }^{* *} P<0,005$; ** $P<0,001$.

\section{Effet du niveau alimentaire sur la teneur plasmatique en leptine}

De manière globale, les concentrations plasmatiques moyennes en leptine (figure 3$)$ ont été plus élevées $(\mathrm{P}<0,05)$ chez les brebis $\mathrm{NH}(3,98 \pm 0,97 \mathrm{ng} / \mathrm{mL})$ que chez les brebis NB $(3,34 \pm 1,17 \mathrm{ng} /$ $\mathrm{mL})$. L'analyse des coefficients de corrélation de Pearson a montré que la teneur plasmatique en leptine était corrélée positivement au poids vif des animaux $(\mathrm{r}=0,78 ; \mathrm{P}<0,001)$, d'une part, et au pourcentage des brebis en chaleurs $(\mathrm{r}=0,68 ; \mathrm{P}<0,05)$, d'autre part.

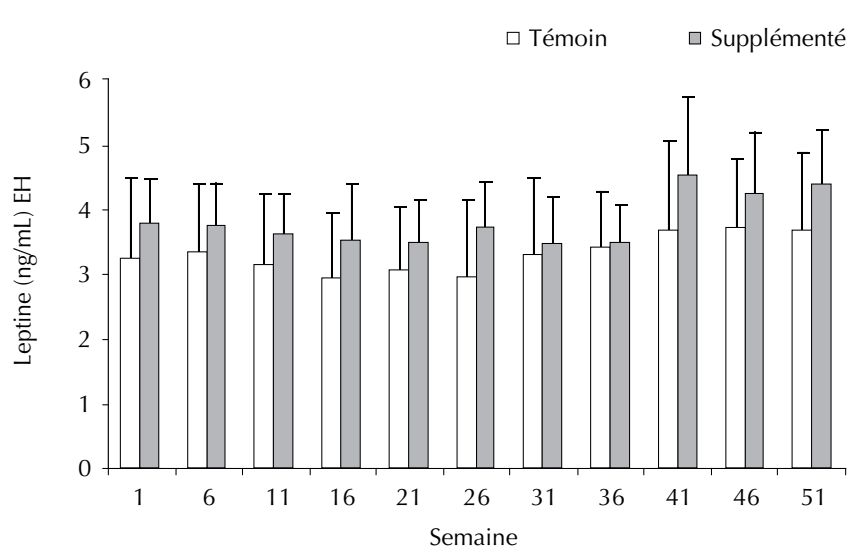

Figure 3 : évolution des teneurs plasmatiques moyennes ( \pm écart-type) en leptine chez les deux lots de brebis en fonction de leur niveau alimentaire bas $(n=9)$ et haut $(n=10)$. EH : équivalent humain. 


\section{Effet du niveau alimentaire sur la teneur plasmatique en IGF-1}

Au début de l'expérience, les deux groupes avaient des concentrations plasmatiques en IGF-1 similaires $(50 \pm 25 \mathrm{ng} / \mathrm{mL}$ vs $47 \pm$ $15 \mathrm{ng} / \mathrm{mL}$; P > 0,05 ; figure 4). Ensuite, durant les vingt-six premières semaines, la moyenne en IGF-1 a été plus élevée chez les brebis NH que chez les brebis NB $(76 \pm 28 \mathrm{ng} / \mathrm{mL}$ vs $58 \pm 21 \mathrm{ng} /$ $\mathrm{mL} ; \mathrm{P}<0,01)$. A partir de la semaine 31 jusqu'à la fin de l'expérience, les teneurs en IGF-1 ont été similaires dans les deux groupes de brebis $\mathrm{NH}$ et $\mathrm{NB}(59 \pm 25 \mathrm{ng} / \mathrm{mL}$ vs $57 \pm 24 \mathrm{ng} / \mathrm{mL}$; $\mathrm{P}>0,05)$. L'analyse des coefficients de corrélation de Pearson a montré que la teneur plasmatique en IGF-1 était corrélée positivement au poids corporel des animaux $(\mathrm{r}=0,30 ; \mathrm{P}<0,05)$.

\section{Effet du niveau alimentaire sur la teneur plasmatique en IGFBP-2 et IGFBP-3}

Les résultats d'analyse des IGFBP ont révélé uniquement la présence d'IGFBP-2 et d'IGFBP-3 avec des taux variables (figures 5 et 6). L'analyse de la variance n'a pas montré de différences signi-

$\square$ Niveau bas $\quad \square$ Niveau haut

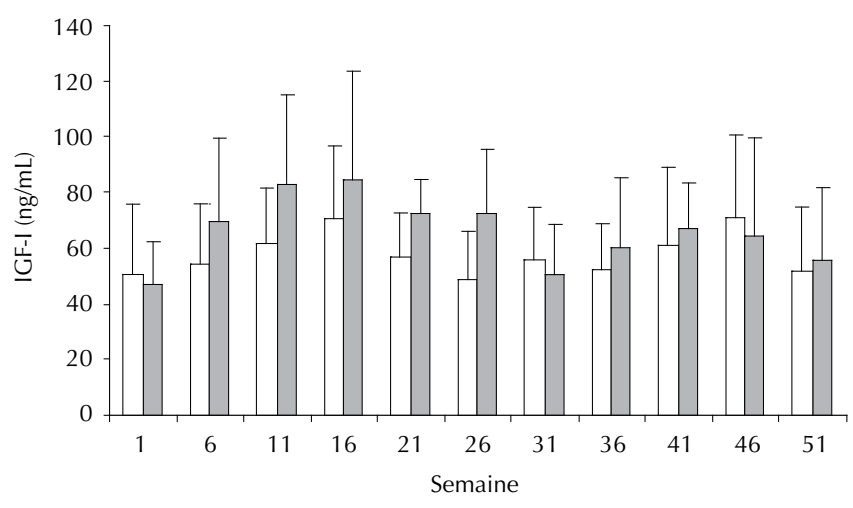

Figure 4 : évolution des teneurs moyennes ( \pm écart-type) plasmatiques de I'IGF-1 chez les deux lots de brebis en fonction du niveau alimentaire bas $(n=9)$ et haut $(n=10)$.

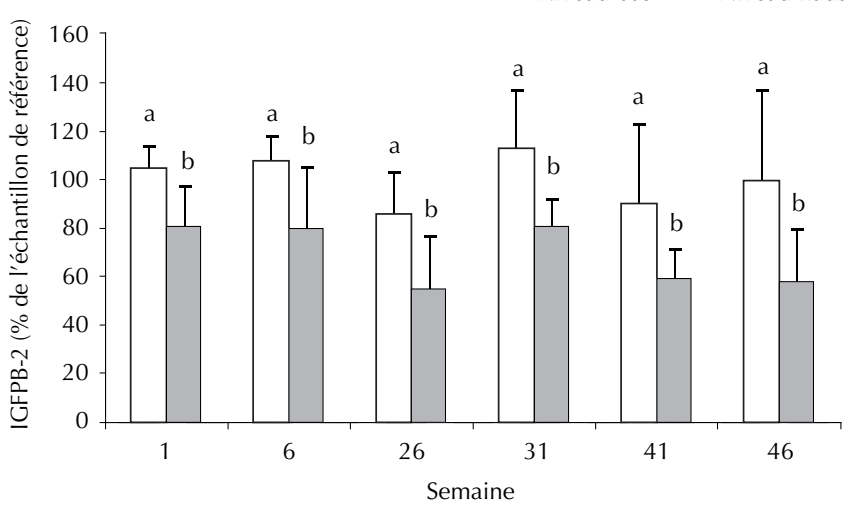

Figure 5 : évolution des teneurs moyennes ( \pm écart-type) plasmatiques des IGFPB-2 chez les brebis à niveau alimentaire bas $(n=9)$ et à niveau alimentaire haut $(n=10)$. Les histogrammes de la même semaine surmontés de lettres différentes représentent des valeurs significativement différentes $(P<0,05)$.

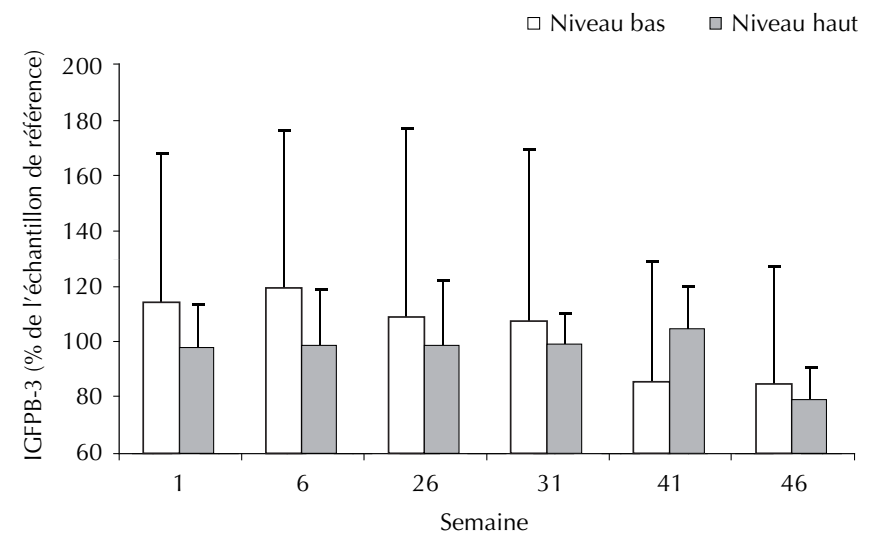

Figure 6 : évolution des teneurs moyennes ( \pm écart-type) plasmatiques des IGFPB-3 chez les brebis à niveau alimentaire bas $(n=9)$ et à niveau alimentaire haut $(n=10)$

ficatives au niveau du taux d'IGFBP-3 entre les deux groupes de brebis, les valeurs étaient en moyenne respectivement de $103 \pm 52$ p. 100 contre $96 \pm 17$ p. $100(\mathrm{P}>0,05)$. En revanche, au niveau de l'IGFBP-2, les brebis ayant reçu un niveau alimentaire bas ont montré des valeurs supérieures à celles des brebis ayant reçu un niveau élevé (en moyenne $99 \pm 24$ p. 100 vs $68 \pm 21$ p. 100 ; $\mathrm{P}<0,01)$. Par ailleurs, l'analyse des coefficients de corrélation de Pearson a révélé une corrélation négative entre la teneur d'IGF-1 et celle d'IGFBP-2 $(\mathrm{r}=-0,41 ; \mathrm{P}<0,05)$.

\section{DISCUSSION}

Ce travail a mis en évidence la saisonnalité de la reproduction chez la race ovine Timahdite dans les conditions naturelles du Maroc, et l'effet déterminant de la photopériode et modérateur du niveau alimentaire. L'activité sexuelle est stimulée par des jours décroissants et inhibée par des jours croissants $(5,6)$. La diminution de la photopériode constitue le signal de stimulation, qui passe par l'augmentation de la synthèse et de la sécrétion de mélatonine et de gonadolibérine (GnRH) (6). Aussi, les présents résultats ont mis en évidence l'effet modérateur de la nutrition déjà décrit par différents auteurs $(11,18)$. L'effet dépressif de la photopériode a été plus prononcé chez les brebis ayant reçu un niveau alimentaire bas que chez celles ayant reçu un niveau alimentaire élevé. Les auteurs ont calculé une corrélation positive entre le pourcentage des brebis en chaleurs ou ayant ovulé, et leur poids corporel.

Ainsi, les brebis NH ont présenté deux fois plus de cycles œstraux normaux par brebis par an que les brebis NB. Des résultats similaires ont été observés chez la chèvre $(24,45)$. Cependant, les deux lots de brebis ont présenté le même nombre moyen de cycles anormaux (ovulations silencieuses ou œstrus anovulatoires) par brebis. Des résultats similaires ont été rapportés chez les ovins et les caprins $(5,22,45)$.

Globalement, les périodes de reproduction définies par cette étude pour la race Timahdite diffèrent de celles rapportées pour la même race (10). Ces différences seraient associées aux variations des conditions d'environnement interannuelles et locales, notamment climatiques et de disponibilité alimentaire.

Les petites élévations d'œstradiol, résultant de la croissance des follicules pendant la transition de la saison d'anœstrus à la saison de reproduction, pourraient expliquer le comportement de l'œstrus anovulatoire observé dans cette étude (5). La présence d'ovulations silencieuses pendant la saison sexuelle, observées aussi chez cer- 
taines races de mouton (29), est difficile à interpréter ; cependant, l'échec de détection des chaleurs ne peut pas être exclu.

La teneur plasmatique en leptine a été corrélée positivement non seulement au poids des animaux, mais aussi à sa variation, confirmant ainsi les résultats d'une étude antérieure (6). D'autre part, cette sécrétion de leptine a également été corrélée significativement au pourcentage des brebis en chaleurs. En effet, la leptine, principalement produite par les adipocytes, peut servir de signal reliant le statut nutritionnel aux performances de reproduction $(6,7)$, puisque des récepteurs de leptine ont été trouvés au niveau de l'hypothalamus et de la glande hypophysaire, indiquant son action à ce niveau (8). Ainsi, des perturbations nutritionnelles entraînent du retard de puberté ou interfèrent avec les cycles œstraux normaux en altérant les fonctions endocrines chez la vache (16) et chez la brebis (13). Des études antérieures ont montré, chez la vache, que l'inhibition de la sécrétion de LH par la restriction alimentaire a été inversée par un traitement avec la leptine concluant à une association positive entre la sécrétion de LH et la leptine (1).

La différence significative qui a été observée entre les niveaux plasmatiques d'IGF-1 des deux groupes de brebis a corroboré les résultats obtenus chez le bovin (9) et a montré que le niveau alimentaire haut a été à l'origine de l'augmentation de la teneur plasmatique en IGF-1. En revanche, la restriction alimentaire a réduit celle-ci. Ceci a été confirmé par la corrélation positive avec le poids corporel des brebis. Des résultats similaires ont été obtenus chez des génisses bien alimentées qui ont produit plus d'IGF-1 que celles ayant eu un déficit alimentaire (44). La réduction systémique de la concentration en IGF-1 après une période prolongée de restriction alimentaire pourrait être expliquée par la diminution des récepteurs hépatiques à la $\mathrm{GH}$ (3).

La biodisponibilité d'IGF-1 est contrôlée par les IGFBP dont les concentrations périphériques sont régulées par la consommation alimentaire (39). La présente étude a montré que les brebis ayant eu un niveau alimentaire bas ont eu des valeurs d'IGFBP-2 supérieures à celles des brebis du niveau haut, résultats concordant avec ceux de Renaville (32) et Massart (21) qui montrent que la restriction alimentaire chez des génisses favorise la synthèse d'IGFBP-2. De même, Renaville et coll. (34) rapportent que la restriction alimentaire résulte généralement en un excès d'IGFBP-2 qui réduit la biodisponibilité d'IGF-1 au niveau des tissus périphériques. Il est supposé que l'augmentation d'énergie alimentaire fait diminuer la concentration d'ARNm codant l'IGFBP-2 et -4 dans les petits follicules antraux, ce qui augmente la biodisponibilité d'IGF-1 et d'IGF-1I dans ces follicules (3, 43). De plus, l'étude a révélé que la teneur en IGF-1 était corrélée négativement à celle en IGFBP-2. Ceci est expliqué par la dégradation de l'IGFBP-2 par la protéine plasmatique placentaire A (PAPP-A) entraînant l'augmentation de la biodisponibilité d'IGF-1 (25). L'expression de l'acide ribonucléique messager (ARNm) de PAPP-A dans les cellules de la granulosa bovine et ovine était maximale dans les follicules préovulatoires et positivement corrélée à l'expression à la fois des récepteurs d'aromatase et de LH (26).

L'analyse de la variance n'a pas montré de différence significative au niveau du taux d'IGFBP-3 entre les deux groupes de brebis $\mathrm{NH}$ et NB. Les effets du niveau alimentaire sur ce paramètre sont controversés. Renaville (32) et Massart (21) rapportent que la restriction alimentaire chez des génisses réduit la synthèse hépatique d'IGF-1 et d'IGFBP-3. De même, Rausch et coll. (31) montrent que la teneur en IGFBP-3 est positivement corrélée à la prise alimentaire. L'augmentation du taux de croissance chez le bovin est associée aux niveaux élevés de l'IGFBP-3 (40). Par ailleurs, des brebis ayant un indice corporel élevé présentent un taux plasmatique d'IGFBP-3 plus important que celles à faible indice corporel (38). D'autres auteurs mettent en évidence chez les ovins (15) et chez les bovins (23) une réduction non significative du taux d'IGFBP-3 après une restriction alimentaire.

\section{CONCLUSION}

Ces résultats confirment la saisonnalité de l'activité reproductrice de la brebis Timahdite pour laquelle la photopériode joue un rôle déterminant. Cette saisonnalité est modulée par le niveau alimentaire. Le fait de doubler les apports nutritionnels d'entretien a permis de prolonger la période d'activité sexuelle et de doubler le nombre de cycles normaux en agissant sur les paramètres hormonaux et métaboliques qui contrôlent les fonctions de la reproduction chez le mouton, en particulier les teneurs plasmatiques en leptine, en IGF-1 et en IGFBP-2.

\section{BIBLIOGRAPHIE}

1. AMSTALDEN M., GARCIA M.R., STANKO R.L., NIZIELSKI S.E., MORRISON C.D., KEISLER D.H., WILLIAMS G.L., 2002. Central infusion of recombinant ovine leptin normalizes plasma insulin and stimulates a novel hypersecretion of luteinizing hormone after short-term fasting in mature beef cows. Biol. Reprod., 66: 1555-1561.

2. ARMSTRONG D.G., GONG J.G., WEBB R., 2003. Interactions between nutrition and ovarian activity in cattle: Physiological, cellular and molecular mechanisms. Reproduction (suppl.), 61: 403-414.

3. ARMSTRONG J.D., COHICK W.S., HARVEY R.W., HEIMER E.P., CAMPBELL R.M., 1993. Effect of feed restriction on serum somatotropin, insulin-like growth factor-1 (IGF-1) and GF binding proteins in cyclic heifers actively immunized against growth hormone releasing factor. Domest. Anim. Endocrinol., 10: 315-324.

4. BISTER J.L., NOEL B., PERRAD B., MANDIKI S.N.M., MBAYAHAGA J. PAQUAY R., 1999. Control of ovarian follicles activity in the ewe. Domest. Anim. Endocrinol., 17: 315-328.
5. BISTER J.L., PAQUAY R., 1983. Fluctuations in the plasma level of the follicle-stimulating hormone during estrous cycle, anestrus, gestation and lactation in the ewe: evidence for an endogenous rhythm of $\mathrm{FSH}$ release. Theriogenology, 19: 565-582.

6. BISTER J.L., PIROTTE C., WERGIFOSSE F., VANDERMEIR M.A., PAQUAY R., 2004. Plasma leptin level in relation with the body weight and its evolution in the ewe. Pflugers Arch Eur. J. Physiol., 447: R1-R12.

7. BISTER J.L., WERGIFOSSE F., PIROTTE C., PIROT P., PAQUAY R., 2004. Leptin and fecundity in ewe. In: Proc. 15th Int. Congr. Animal Reproduction, Porto Seguro, Brazil, Aug. 2004, 316 p.

8. BJORBAEK C., KAHN B.B., 2004. Leptin signaling in the central nervous system and the periphery. Recent Prog. Horm. Res. 59: 305-331.

9. BOSSIS I., WETTEMAN R.P., WELTY S.D., VIZCARRA J.A., SPICER L.J., DISKIN M.G., 1999. Nutritionally induced anovulation in beef heifers: ovarian and endocrine function preceding cessation of ovulation. J. Anim. Sci., 77: 1536-1546. 
10. BOUJENANE I., 1999. Les ressources génétiques ovines au Maroc. Rabat, Maroc, Actes Edition, 136 p.

11. BUTLER W.R., 2000. Nutritional interactions with reproductive performance in dairy cattle. Anim. Prod. Sci., 60-61: 449-457.

12. CHEMINEAU P., DAVEAU A., MAURICE F., DELGADILLO J.A., 1992. Seasonality of estrus and ovulation is not modified by subjecting female alpine goats to a tropical photoperiod. Small Ruminant Res., 8: 299-312.

13. ESTIENNE M.J., SCHILLO K.K., HILEMAN S.M., GREEN M.A., HAYES S.H., BOLING J.A., 1990. Effects of free fatty acids on luteinizing hormone and growth hormone secretion in ovariectomized lambs. Endocrinology, 126: 1934-1940.

14. FORTUNE J.E., RIVERA G.M., EVANS A.C., TURZILLO A.M., 2001 Differentiation of dominant versus subordinate follicles in cattle. Biol. Reprod., 65: 648-654.

15. GALLAHER B.W, BREIER B, OLIVIER M.H, HADING JE GLUCKMAN P.D., 1992. Ontogenic differences in the nutritional regulation of circulating IGF binding proteins in sheep plasma. Acta Endocrinol., 126: 49-54.

16. HOUSEKNECHT K.L., BOGGS D.L., CAMPION D.R., SARTIN J.L., KISER T.E., RAMPACEK G.B., AMOS H.E., 1988. Effect of dietary energy source and level on serum gorwth hormone, insulin-like growth factor-1, growth and body composition in beef heifers. J. Anim. Sci., 66: 29162923

17. INRA, 1988. Tables de I'alimentation des bovins, ovins et caprins Paris, France, Inra, $192 \mathrm{p}$.

18. LUCY M.C., 2003. Mechanisms linking nutrition and reproduction in postpartum cows. Reprod. domest. Ruminants, 61: 415-417.

19. MALPAUX B., VIGUIE C., THIERY J.C., CHEMINEAU P., 1996. Contrôle photopériodique de la reproduction. Prod. Anim., 9 : 9-23.

20. MANI A.U., MCKELVEY W.A.C., WATSON E.D., 1996. Effect of undernutrition on gonadotropin profiles in non-pregnant, cycling goats. Anim. Reprod. Sci., 43: 25-33.

21. MASSART S., 2000. Caractérisation et significations biologiques des "Insulin-like growth factors-binding proteins» chez les bovins. Thèse Doc., FUSAGx, Gembloux, Belgique, 146 p.

22. MBAYAHAGA J., MANDIKI S.N.M., BISTER J.L., PAQAY R., 1998. Body weight, oestrus and ovarian activity in local Burundian ewes and goats after parturition in the dry season. Anim. Reprod. Sci., 51: 289-300.

23. MCGUIRE M.A., DWYER D.A., HARRELL R.J., BAUMAN D.E., 1995 Insulin regulates insulin-like growth factors and some of their binding proteins in lactating cows. Am. J. Physiol. Endocrinol. Metab., 269: E723E730.

24. MELLADO M., FOOTE R.H., GOMEZ A., 1991. Reproductive efficiency of Nubian goats throughout the year in Northern Mexico. Small Rum. Res. 6: 151-157.

25. MONGET P., FABRE S., MULSANT P., LECERF F., ELSEN J.M., MAZERBOURG S., PISSELET C., MONNIAUX D., 2002. Regulation of ovarian folliculogenesis by IGF and BMP system in domestic animals. Domest. Anim. Endocrinol., 23: 139-154.

26. MONGET P., MAZERBOURG S., DELPUECH T., MAUREL M. C. MANIERE S., ZAPF J., LALMANACH G., OXVIG C., OVERGAARD M.T., 2003. Pregnancy-associated plasma protein A is involved in insulin-like growth factor binding protein-2 (IGFBP-2) proteolytic degradation in bovine and porcine preovulatory follicles: identification of cleavage site and characterization of IGFBP-2 degradation. Biol. Reprod., 68: 77-86.

27. MONGET P., MONNIAUX D., 1995. Growth factors and the control of folliculogenesis. J. Reprod. Fertil. (suppl.), 49: 321-333.

28. NOEL B., PERRAD B., MANDIKI S.N.M., BISTER J.L., PAQUAY R., 1998. Effects of IGF-1 and EGF on steroidogenesis and sensitivity to LHFSH of entire dominant follicles perifused in vitro. In : Actes $5^{\mathrm{e}}$ Renc. Rech. Ruminants, Paris, France, déc. 1998, 49 p.

29. ORTAVANT R., BOCQUIER F., PELLETIER J., RAVAULT J.P., THIMONIER J., VOLLAND-NAIL P., 1988. Seasonality of reproduction in sheep and its control by photoperiod. Aust. J. Biol. Sci. 41: 69-85.
30. PARKER A., GOCKERMAN A., BUSBY W.H., ClEMMONS D.R., 1995. Properties of an insulin-like growth factor binding protein-4 protease that is secreted by smooth muscle cells. Endocrinology, 136: 2470-2476.

31. RAUSCH M.I., TRIPP M.W., GOVONI K.E., ZANG W., WEBER W.J., CROOKER B.A., HOAGLAND T.A., ZINN S.A., 2002. The influence of level of feeding on growth and serum insulin-like growth factor 1 and insulin-like growth factor-binding proteins in growing beef cattle supplied with somatotropin. J. Anim. Sci., 80: 94-100.

32. RENAVILLE R., 1997. L'axe somatotrope et les productions bovines. Contribution à la compréhension de certains mécanismes impliqués dans la croissance et la lactation. Thèse Agrégé Enseignement supérieur, FUSAGx, Gembloux, Belgique, $142 \mathrm{p}$.

33. RENAVILLE R., MASSART S., SNEYERS M., FALAKI M., GENGLER N., BURNY A., PORTETELLE D., 1996. Dissociation of increases in plasma insulin-like growth factor 1 and testosterone during the onset of puberty in bulls. J. Reprod. Fertil., 106: 79-86.

34. RENAVILLE R., VAN EENAEME C., BREIER B.H., VLEURICK L., BERTOZZI C., GENGLER N., HORNICK J.-L., PARMENTIER I., ISTASSE L., HAEZEBROECK V., MASSART S., PORTETELLE D., 2000. Feed restriction in young bulls alters the onset of puberty in relationship with plasma insulin-like growth factor-1 (IGF-1) and IGF-binding protein. Domest. Anim. Endocrinol., 18: 165-176.

35. RIVERA G.M., ALANIS G.A., CHAVES M.A., FERRERO S.B., MORELLO H.H., 2003. Seasonality of estrus and ovulation in Creole goats of Argentina. Small Rumin. Res., 48: 109-117.

36. SAS, 1991. SAS user's guide, Vers. 6. Cary, NC, USA, SAS Institute.

37. SCARAMUZZI R.J., MURRAY J.F., DOWNING J.A., CAMPBELL B.K., 1999. The effects of exogenous growth hormone on follicular steroid secretion and ovulation rate in sheep. Domestic. Anim. Endocrinol., 17: 269-277.

38. SNYDER J.L., CLAPPER J.A., ROBERTS A.J., SANSON D.W., HAMERNIK D.L., MOSS G.E., 1999. Insulin-like growth factor-1, insulin-like growth factor binding proteins and gonadotropins in the hypothalamic-pituitary axis and serum of nutrient restricted ewes. Biol. Reprod., 61: 219-224.

39. THISSEN J.P., KETELSLEGERS J.M., UNDERWOOD L.E., 1994. Nutritional regulation of the insulin-like growth-factors. Endocr. Rev., 15: 80-101.

40. VESTERGAARD M., PURUP S., HENCKEL P., TONNER E., FLINT D.J., JENSEN L.R., SERJRSEN K., 1995. Effects of growth hormone and ovariectomy on performance, serum hormones, insulin-like growth factor binding proteins, and muscle fibre properties of prepubertal Friesian heifers. J. Anim. Sci., 73: 3574-3584.

41. WEBB R., GARNSWORTHY P.C., GONG J.-G., ARMSTRONG D.G., 2004. Control of follicular growth: Local interactions and nutritional influences. J. Anim. Sci., 82: E63-E74.

42. WEBB R., GARNSWORTHY P.C., GONG J., ROBINSON R.S., WATHES D.C., 1998. Consequences for reproductive function of metabolic adaptation to load. In: Metabolic stress in dairy cows. Penicuik, UK, British Society of Animal Science, 10 p.

43. WEBB R., NICHOLAS B., GONG J.G., CAMPBELL B.K., GUTIERREZ C.G., GARVERICK H.A., ARMSTRONG D.G., 2003. Mechanism regulating follicular development and selection of the dominant follicle. Reprod. domest. Ruinants, 61: 71-90.

44. YUNG M.C., VANDER HAAR M.J., FOGWELL R.L., SHARMA B.K., 1996. Effect of energy balance and somatotropin on insulin-like growth factor- 1 in serum and on weight and progesterone of corpus luteum in heifers. J. Anim. Sci., 74: 2239-2244.

45. ZARAZAGA L.A., GUZMAN J.L., DOMINGUEZ C., PEREZ M.C., PRIETO R., 2005. Effect of plane of nutrition on seasonality of reproduction in Spanish Payoya goats. Anim. Reprod. Sci., 87: 253-267.

Accepté le 18.03.2010 


\section{Summary}

Archa B., Chentouf M., Bister J.L. Effect of the Feed Level on the Seasonality of the Sexual Activity of Timahdite Ewes: Role of the Leptin and IGF System

The effects of the feed level on the seasonality of the sexual activity were studied in Timahdite ewes in the Middle Atlas region of Morocco $\left(33^{\circ} \mathrm{N}\right)$. Non pregnant and non lactating adult ewes (3-4 years) were allocated to two groups according to two diet levels: $\mathrm{HL}(\mathrm{n}=10)$ received double the maintenance requirements and $L L(n=9)$ the maintenance requirements. Over a one-year period, estrus was recorded twice daily and blood samples were collected weekly to determine progesterone, leptin, IGF-1, IGFBP-2 and IGFBP-3 plasmatic levels. The higher feed level stimulated the reproductive activity in HL ewes, which presented a longer reproductive season and a significantly higher number of normal estrous cycles than LL ewes $(7.8 \pm 1.4$ vs $3.9 \pm 1.9$, respectively; $\mathrm{P}<0.05)$. Leptin, IGF-1 and IGFBP-2 seemed involved in the stimulation of the reproductive activity. Compared with LL ewes, HL ewes showed significantly higher plasmatic levels in leptin (3.79 \pm 0.38 vs $3.30 \pm 0.29 \mathrm{ng} / \mathrm{mL} ; \mathrm{P}<0.05)$ and in IGF-1 $(76 \pm 28$ vs $58 \pm 21 \mathrm{ng} / \mathrm{mL} ; \mathrm{P}<0.01)$, and a significantly lower level in IGFBP-2 $(68 \pm 21 \%$ vs $99 \pm 24 \%$; $P<0.01)$. No significant difference was recorded between the two groups regarding the IGFBP-3 levels $(103 \pm 52 \%$ vs $96 \pm 17 \%$; P > 0.05). These results showed that the Timahdite ewe exhibited a seasonal reproductive pattern which was modulated by the feed level.

Keywords: Sheep - Feeding level - Reproduction - Periodicity Season - Blood protein - Insulin-like growth factor - Morocco.

\section{Resumen}

Archa B., Chentouf M., Bister J.L. Efectos del nivel alimenticio sobre la estacionalidad de la actividad sexual en la oveja Timahdite: influencia de la leptina y del sistema IGF

Se estudiaron los efectos del nivel alimenticio sobre la estacionalidad de la reproducción en la oveja Timahdite en la región del Atlas medio en Marruecos $\left(33^{\circ} \mathrm{N}\right)$. Se repartieron ovejas adultas (3-4 años), no grávidas y no lactantes, en dos lotes $\mathrm{NH}(\mathrm{n}=10)$ y $\mathrm{NB}(\mathrm{n}=9)$, en función de los regímenes alimenticios correspondiendo respectivamente a dos y una veces las necesidades de mantenimiento. Durante un año, se observaron calores dos veces por día y se realizaron tomas de sangre semanales para la dosificación de los niveles plasmáticos de progesterona y de factores de crecimiento como leptina, IGF-I y sus proteínas de unión (IGFBP-2 et IGFBP-3). El nivel alimenticio elevado estimuló la función reproductora de las ovejas $\mathrm{NH}$, debido a que estas presentaron una estación de reproducción más larga y manifestaron un número de ciclos normales significativamente más elevado que las ovejas del lote NB $(7,8 \pm 1,4$ vs 3,9 $\pm 1,9 ; \mathrm{P}<0,05)$. La leptina, la IGF-I y la IGFBP-2 parecieron implicadas en esta estimulación de la actividad reproductiva. Las ovejas $\mathrm{NH}$ presentaron contenidos plasmáticos significativamente más elevados que las ovejas NB en leptina $(3,79 \pm 0,38$ vs $3,30 \pm$ $0,29 \mathrm{ng} / \mathrm{mL} ; \mathrm{P}<0,05)$ y en IGF-I $(76 \pm 28$ vs $58 \pm 21 \mathrm{ng} / \mathrm{mL}$, $\mathrm{P}<0,01$ ), mientras que el contenido plasmático en IGFBP-2 fue significativamente más elevado en las ovejas NB $(99 \pm$ 24 vs $68 \pm 21$ p. $100, \mathrm{P}<0,01)$. En cambio, no se observó ninguna diferencia significativa para el contenido de IGFBP-3 $(103 \pm 52$ vs $96 \pm 17$ p. $100, \mathrm{P}>0,05)$. El presente trabajo permitió concluir que la oveja Timahdite presentó una actividad reproductiva estacional modulada por la alimentación.

Palabras clave: Ovino - Nivel alimenticio - Reproducción Periodicidad - Estación del año - Proteína sanguínea - Factor de crecimiento - Marruecos. 